\title{
Improving Dental Access and Oral Health of Older Adults Living in Residential Care: A Qualitative Research
}

\author{
Samantha Glover ${ }^{1}$ Russell Kabir ${ }^{2}$ \\ ${ }^{1}$ Dental Public Health Program Manager, Public Health England, \\ East of England, United Kingdom \\ ${ }^{2}$ School of Allied Health, Anglia Ruskin University, United Kingdom
}

Address for correspondence Russell Kabir, PhD, School of Allied Health, Anglia Ruskin University, United Kingdom (e-mail: russell. kabir@aru.ac.uk).

Eur J Gen Dent 2021;10:84-90.

\begin{abstract}
Objectives To explore the access to daily oral care and access to dentistry for those living in a residential care home.

Materials and Methods For this research, a residential care home and a dental practice within the local area to the care home was approached. A total of 45 participants were interviewed.

Statistical Analysis Semi-structured interviews with three separate cohorts were performed, the patients or their friends and families, care home staff, and the dental teams. The data were analyzed using thematic analysis.

Results The three cohorts of patients had similar concerns in accessing the dentists

Keywords

- oral health

- residential care

- qualitative

- dental access

- older adults including transport, communication, training, and the need for raised awareness of the importance of oral health. The access to daily oral care was impacted upon by apathy and lack of education or training.

Conclusion Better training is required for care home staff and older people living in care on the importance of oral health. The provision of dental services for this community could be delivered differently with a focus on preventing and maintaining independence as long as possible.
\end{abstract}

\section{Introduction}

There is an ever-increasing population of people aged over 65 years and an increasing number living beyond 85 years. Although many are staying well into older age and living independently, there is an estimated 400,000 living in residential care homes. ${ }^{1}$ The population of older people living in Essex is increasing faster than the national average with
Thurrock and Southend having a significantly higher percentage of people over 65 years permanently living in care than the national average. ${ }^{2}$ Further attention to the impacts of oral health for those living in care homes was highlighted by the recent Care Quality Commission (CQC) report that highlighted access to routine dental care and oral health care plans as areas of concern. ${ }^{1}$
DOI https://doi.org/ $10.1055 / \mathrm{s}-0041-1735764$ ISSN 2320-4753
(C) 2021. European Journal of General Dentistry.

This is an open access article published by Thieme under the terms of the Creative Commons Attribution-NonDerivative-NonCommercial-License, permitting copying and reproduction so long as the original work is given appropriate credit. Contents may not be used for commercial purposes, or adapted, remixed, transformed or built upon. (https://creativecommons.org/licenses/by-nc-nd/4.0/).

Thieme Medical and Scientific Publishers Pvt. Ltd. A-12, 2nd Floor, Sector 2, Noida-201301 UP, India 
There are 22,000 people living with dementia in the Essex, Southend, and Thurrock areas, which is predicted to increase to over 35,000 by $2035 .^{3}$ Currently in the United Kingdom (UK), there are approximately 850,000 or 1 in 6 people living with dementia (data from 2015). With this large and growing cohort of people living with dementia, services must adjust to accommodate them and help support carers. Advances have been made across the National Health Service (NHS) and social care strategically since 2009 when the national dementia strategy was published. However, oral health and links with dental services received less attention. ${ }^{4}$ There are now recognized pathways published by the National Institute for Clinical Excellence (NICE) in oral health, including 5

- Polices in place on oral health that provide the residents with support to access dental services.

- Undertake oral health assessments and mouth care plans.

- Daily mouth care and keeping records.

- To update care staff knowledge and skills.

Dental practitioners are to:

- Provide residents in care homes with routine and specialist preventive care and treatment as necessary, in line with local arrangements.

- Ensure dentures are appropriately marked with a name.

As health and care have improved, people are living longer. Improvements in oral health and oral healthcare have resulted in people usually retaining at least some natural teeth for life. Dental service providers now report increased numbers of older patients who require complex dental treatment as their dentition is already heavily restored. Treatment needs are often complicated further by acute and chronic medical conditions that become more common with increasing age. Recent research has further confirmed associations between poor oral health and certain medical conditions, for example, aspiration pneumonia, and medical conditions such as dementia are associated with poor oral hygiene. ${ }^{6}$ Maintaining day-to-day oral health standards to preserve oral health as we age is crucial for everyone, and it should be recognized that even at very early stages of dementia, oral health routines may slip, with patients requiring extra help and supervision as part of day-to-day social and personal care routines. Academic and clinical research has been performed on people living in care homes with or with dementia focusing on different interventions to improve short-term oral health. These have ranged from clinical interventions, such as fluoride application and hygienists going to care homes to brush teeth, to more educational routes such as comprehensive training for care staff on carrying out daily oral hygiene. ${ }^{7,8}$ Essex has its own dementia care strategy, and this was used as a basis to design a flowchart and identify the current provision and potential pathway for adults in care to access dentistry. ${ }^{9}$ Local dementia care pathways should include both day-to-day maintenance of oral health plus access to mainstream dental services that have reasonable adjustments to accommodate people affected by dementia and their careers. This study differs from these by looking at the potential barriers and opportunities from a qualitative perspective of the three different users of the pathway; the resident, care staff, and dental team.

\section{Materials and Methods}

\section{Study Settings}

Initial contact was made with a large care home based in Southend on the sea. This 60-bed care home was approached as they had a variety of residents including those requiring nursing care, those living with dementia, and many who required limited care needs. It is part of a chain of care homes, Hallmark Care, and had a good CQC rating and good links into the local community. The care home was keen to take part and felt a need for better knowledge and an improved pathway for oral health that would benefit the staff and residents. A dental practice within the local area to the care home was approached to take part in the research, as well as the practicalities of this being a local dental service that the residents of the care home could access. It was a well-established practice with a range of dental care professionals and had a positive CQC inspection with no action required in any areas.

\section{Sample Selection}

To gather a range of views, the sample was selected to include staff in both the care home and dental practice of different grades or job roles as shown in - Table $\mathbf{1}$.

Two training sessions were delivered to the staff at the care home to give them an understanding of the importance of oral health and hygiene as well as some practical guidance on how to support others with daily hygiene routines. This also helped engage the care home in the research program. All information delivered was based on the principles outlined in Delivering Better Oral Health. ${ }^{10}$

\section{Data Collection and Analysis}

Semi-structured individual interviews were chosen as the preferred research method to create a safe space where the participants could share their views without the influence of hierarchy or fear of unfavorable treatment. Other previous studies in this area had used questionnaires and used a combined quantitative and qualitative methodology, during this study it was agreed that individual interviews would allow for a fuller explanation of the answers given, allowing people to elaborate and provide a richer source of data. A range of participants from each cohort group was recruited to try and gain and diverse a response as possible, this included seeking out different grades and skill levels of both care home and dental staff, these participants' characteristics are recorded in - Table $\mathbf{1}$. The interviews were recorded using a dictaphone and transcribed afterward. A thematic analysis of these transcripts was performed by the primary researcher and the themes were shared with the participants for quality assurance. These themes would then be dissected and compared against the other cohorts (resident, care professional, dental care professional) to identify any cross-cutting 
Table 1 Participants' job role and gender

\begin{tabular}{|l|l|l|l|}
\hline Staff grade & Male & Female & Subtotal \\
\hline Senior & & 3 & 3 \\
\hline Manager & 1 & 1 & 2 \\
\hline HCA & & 2 & 2 \\
\hline Dental practice & & & \\
\hline Dentist & 1 & 1 & 2 \\
\hline Nurse & & 1 & 1 \\
\hline $\begin{array}{l}\text { Receptionist } \\
\text { Resident/friends and } \\
\text { family }\end{array}$ & 16 & 19 & 35 \\
\hline Total & & & 45 \\
\hline
\end{tabular}

or contradicting themes. A second analysis was factored in, the coded transcripts were shared with senior dental public health professionals to ensure the same themes were identified. A content analysis approach was used to highlight frequently occurring themes but also linking these to the context in which they were shared and the impacting variables, for example, the participant's position, such as job role. ${ }^{11}$ Although the questions were answered anonymously, it was possible to identify who gave which information due to the small number of participants taking part in each group.

\section{Ethical Approval}

The Public Health England approval process was followed with support from the Head of Research Governance, Research Translation \& Innovation Division. After consulting the Public Health England Research team, it was decided that full National Institute for Health Research (NIHR) approval was required due to the potential vulnerability of some of the participants, the requested forms and documentation were sent. After the presentation and the Social care REC and minor amendments being made to the supporting documentation, consent forms, and patient information sheets, approval was granted. A consent form, patient participation sheet, and questions were guide-created for each cohort group for the research and shared with each participant. This supports the researcher and the participant in understanding the process of the interview to achieve the desired purpose. ${ }^{11}$

\section{Findings}

The thematic analysis highlights several reoccurring themes across the three cohort groups and they are:

- Transport and access

- Motivation and apathy

- Training

- Communication

- Finance

\section{Themes 1: Transport and Access}

The most common theme identified by every cohort group was the transport to access the dental provision, this could be further impacted by the mobility issues of the patients such as requiring a wheelchair, accessible taxi, or adapted transport. One carer gave the example of a patient who had received a fairly positive dental visit but had waited for transport home afterward, leading to the resident having to be locked in the practice for some time while they waited for the taxi to collect them. Transport was an issue raised by family members too either accompanied by a paid carer or not; the uncertainty over waiting times at the dentist and the availability of transport facility of patients were concerns.

"waiting for transport afterwards, they had to lock the door ... because she kept wanting to go" (Care home professional)

"when you go to the dentist you could be in the waiting room 40 mins" (friends and family)

"I have had to take over the driving, I don't like it but otherwise we couldn't get there" (friends and family)

Parking was also raised as an issue for accessing dental care, if a carer had arranged transport it was often difficult to park or drop the patient off at the practice even if parking was allocated.

"we have two spaces ... we try to keep free for disabled patients, but it doesn't always work" (dental care professional)

The frustration over transport was highlighted by the dental team who often found patients from care homes arriving late or completely missing appointments due to transport issues. The suggestion of domiciliary care to overcome this issue was raised to get over this barrier but this service, in Essex, is currently only available for house-bound or bed-bound patients who are in an acute situation such as pain or lost denture as opposed to routine dental care. This reinforces the message that this cohort of patients received reactive rather than preventive care. A dental professional also suggested domiciliary dentistry as the last resort.

"during a domiciliary visit, the dental care professional is in a compromised position ... it's not practical and there is a lot of equipment you need to take with you and it is not always possible to ... it also limits your diagnostics such as X-rays" (dental care professional)

“... actually carrying out the treatment such as fillings can be difficult as she [the patient] cannot move so as the dental team you have to stand in an awkward position as does the nurse in order to carry out the treatment" (dental care professionals) 
"... a home visit they either tend to be on a waiting list or some dentist don't do it" (care home professional)

"Two of our dentists here do home visits ... especially for long standing patients" (dental care professional)

Most of the care home professionals wanted to have a dentist come to the home to carry out routine dental care but had experienced delays when trying to access dental care and had felt when the dentist did come out they were quite rushed, despite this, they still felt it was the best solution for the resident.

"if they are in their own environment it is probably a bit more comfortable for them" (care home professional)

Some carers spoke about not only the issue with physical transport but how they as the professional could feel vulnerable taking a resident outside of the safety of the care home on their own.

"it's less dauting to the carer to be in their own environment then taking someone out because you know there could be an accident, or they may have a bad reaction to the injection they have to have for their teeth or whatever" (care home professional)

This raised another interesting challenge over the relationship between the care home and dental practice, the dental teams felt that once the patient was in the practice they were under their care and were very qualified professionals, but this was not the perspective of the carers. Those family members who took residents to the dentist felt very safe in the care of the professionals there. The frustration over transport was echoed by family members also who found that getting someone to the dentist was another expense to be considered and sometimes the journey could be more daunting than the treatment. Those residents who accessed dentistry with a family member were conscious that if they were not driven there by them, they would not able to attend.

\section{Themes 2: Motivation and Apathy}

The importance of oral hygiene was raised by each group who felt that care staff and those living in residential care did not see teeth as important. The disparity between physical care and oral care was raised by several participants.

"It's so difficult to get him there and I have so many other things I have to do like doctors and hospitals and everything. It is just not a priority, I can't do everything" (friends and family)

Routinely, residents were asked who their general practitioner was but not usually the gross domestic product (GDP), and if one was not declared there was no follow-up action in looking for a GDP. Yet, when speaking to senior members of staff, it was identified that the question about having a dentist was asked at several stages and recorded in the residents' notes.

"when I take the initial enquiry I have to ask about dentist, dentistry, food habits, eating habits ..." (care home professional)

"it should be right there on the front of the care plan really, the dentist detail next to the doctors' details so you can see it straight away as an immediate contact' (Care home professional)

It was expressed by family members and carers alike that there was an apathy amongst some residents that they were waiting to die so what is the point, or in the greater scheme of things it did not matter.

"it's something that gets moved to the back burner really" (friends and family)

It was felt by some that this apathy came from the staff themselves and that both staff and residents alike did not see the importance of oral care and accessed service only when they had a problem.

"if care staff do not have a very good oral health regime they cannot pass this on to the residents" (dental care Professional)

Others commented on the decline of personal oral health after a hospital stay.

"throughout that her teeth were neglected without a doubt" (friends and family)

There was an apparent disparity, highlighted by the dental professionals, between those living with family members and those living in care, for those cared for by a family member at home it seemed that dental appointments and daily oral hygiene were maintained, but this slipped and became less important when they went to a care home setting.

"if they have family and they are living at home, it never seems to be a problem" (dental care professional)

Some carers commented on the difficulty faced in carrying out personal care, with some residents refusing to have their teeth brushed or becoming violent or aggressive during brushing. Others commented on the apathy that some residents felt and how this impacted their motivation to carry out daily oral care.

"what's the point, you're here to die and its only your teeth" (care home professional) 
The apathy around dental care was not always intentional, during a conversation with a family member, the researcher was told about how a daughter regularly took her mother out of the care home to visit her hairdresser and for lunch. When asked if she took her to the dentist, she said she had not thought about it and her mother did not have a toothache. Some family members assumed this was part of routine care in the care home and others just did not think about it until it was raised by someone else or their family member had pain.

\section{Theme 3: Training}

The dental professional felt that they had adequate training in supporting a range of individuals with additional needs, the practice involved in the study had received dementia friends training. Two of the suggestions were that the dental teams treated everybody as an individual with a specific set of needs and that they were required to complete continuing professional development (CPD) to maintain their professional registration, which ensured they were looking at current training and keeping up to date with the latest evidence, guidance, and legislation. The only area that was expressed as lacking by the dental community was specific support for those living with dementia. This was an increasing demand, with more and more patients accessing services with early dementia. But this was not mirrored by a relative of a care home resident who felt that the staff at the practice she took her mother to had the understanding and expertise to support someone with dementia.

"I don't think the dentist was adequately trained, not at all" (friends and family)

This was not the view of all family members, several expressed really positive experiences with "younger dentists" and wondered if they were now taught about these things during their training. They also spoke of the community support that was available in the area and how the local dementia teams wanted to go to train the dental teams, but they were too busy.

"training would give the carers more confidence [in delivering oral care]" (care home professional)

"[care home staff] are often understaffed and very stretched, but I don't think they have adequate training on how to support people's oral health, they sometimes seem nervous about brushing people's teeth, taking dentures in or out and checking the mouth" (Dental care professional)

All of the care home professionals felt they required more training, some had previously had training but felt it was not sufficient. They felt that the delivery of the training was important and that face-to-face worked best. Who delivered the training was also highlighted by one of the care professionals who felt that the residents and care home staff would appreciate training coming from a dental care professional rather than a care home trainer or other members of staff.
"I've done it [online learning] and flicked through it, guessed the questions at the end, it's not training" (care home professional)

"It's all well and good watching a power point and having someone chat [...] but unless you actually do it you don't know ..." (care home professional)

The dental professionals also felt that the care home staff would benefit from additional oral care training.

"A dental nurse could go in to a care home ... have face to face training with brushing demonstrations and having a go ... there are some examples of e-learning programs that could be another option" (dental care professional)

The training on the importance of oral health was also highlighted as a need for those residential residents who still had capacity and the ability to carry out their own oral care.

"my residential resident and people like that it would be fantastic for them" (care home professional)

\section{Theme 4: Communication}

It was expressed by the dental community that communication was a concern when it came to contacting patients living in care homes. GDPR may have an impact on this but it was felt that when a phone call was made to a care home by the dental receptionist it may not always get through to the patient or their next of kin. There was also a concern raised over communicating with others and the carers' understanding of the consent process, some were happy for carers to be spoken to directly and to be involved in the decision, but this still required consent.

“when you call a care home you don't know who you are talking to" (dental care professional)

"support staff do not always understand the importance of the paperwork ... or the consent process... [this] can delay the patients treatment" (dental care professional)

Communication of the patients' needs and requirements could also pose a problem for dental staff who may not have adequate information to treat the patient or have sufficient information when booking the appointment to put reasonable adjustments in place such as booking a downstairs clinic for patients with mobility issues. The power of attorney was also not thought to be fully understood by care home professionals.

"it's when you don't know, and they just turn up, that hard" (dental care professional) 
Concerns over the patient understanding or retaining information were important and those with a family member who could attend appointments mentioned how important it was that they went into the room with their family members as even those without dementia often forgot or got confused over what the dental professional had said.

"We go in the car and my wife comes in with me" (resident with family member)

Communication and discussion around oral care and dental health, in general, was also raised by several of the care home staff who felt that general health and personal care were talked about much more than dental care. It was felt that the impacts of poor oral health were not understood by those living in care or carers.

"I think in terms of oral care it needs to be more out there, it is not out there to be honest whether in hospitals, care homes or anywhere" (care home professional)

\section{Theme 5: Finance}

Another barrier raised by the care home team was funding, this was not only about the cost of dental treatment but also not holding the funds or having adequate information on who was entitled to free dental treatment and the exemption evidence required. Finance was a secondary issue for those paying for dental treatment and also having to pay for carers to accompany them to appointments and the cost of transport to and from the dental appointment.

"we charge for a carer to take them and it is then a stumbling block because that can be quite expensive" (care home professional)

One resident had been paying for a dental care plan for over a year but was unable to go due to limited access in the practice and the resident's poor mobility. The expense of treatment or the perceived cost of treatment was not well understood. Many family members were confused about who's responsibility it was or if a patient with additional needs could still see a "normal" dentist. Little was known about who was entitled to free dental treatment or the costs of treatment. Many of those who took family members to the dentist accessed private dental services.

This was also raised by the dental profession as frustration over not knowing if the patient pays for dental treatment or not and not having the adequate funds or exemption evidence with them. There was also a disparity in the dental care available to those who can afford dental treatment and those who cannot, very few people were entitled to free dental care, and the difference in the availability of services between those who can afford to pay for private dental service and those who cannot was clear. "the NHS do not offer [home visits] as a service, it is something that has to be paid for" (dental care professional)

Most people receive NHS dental care through General Dental Services. Community Dental Services is an additional service, commissioned by NHS England to serve the most vulnerable population groups. This is a specialized referral-based service for people who cannot access general dental services. In Essex, these services include domiciliary dental care where members of the dental team can visit a patient who is housebound, to provide support to keep a patient out of dental pain, not for routine dental care. Community dental providers report that they are often called upon to deal with problems that would have been prevented had the patient been receiving regular dental care and had better standards of day-to-day mouthcare.

\section{Discussion}

It was the intention of the research to have equal inclusion from across the three cohort groups, but certain groups were very difficult to recruit. Staff in the care home were reluctant to give consent or suggest certain residents who would be willing and happy to be interviewed and would have the capacity to consent from themselves. Getting the family members into the care home was also a challenge. This resulted in more care home staff and dental professionals being interviewed. To overcome this, a focus group was arranged with the support of the dementia team that included those living with dementia and their family members. One of the care home professionals interviewed described a model she previously used in a care home she worked in, which had a partnership arrangement with two local dental practices. The patients were registered with the local practice when they first came to the care home and this practice took over their routine care. The relationship was mutually supported with the care home being able to call the practice for ad-hoc support and advice as well as regular routine dental visits and emergency visits to the care home, this was in the Walthamstow area but the participant could not remember the practice name. Several care home staff talked about Activities of Daily Living (ADLs), which are basic tasks that must be accomplished every day for an individual to thrive. Generally, ADLs can be broken down into the following categories:

- Personal hygiene, including bathing, grooming, oral care, nails and hair care.

- Continence management.

- Feeding.

- Ambulating.

- Dressing.

These are regularly recorded and monitored, and some referred to them as a place where oral care is recorded, or notes are made about the resident's ability to carry out basic tooth brushing, one carer went on to say how this should be expanded to have a tick sheet such as she had in a previous 
home to record if this had been done. Daily oral health care charts are a resource recommended for every resident, with or without capacity, to brush their teeth. They can become a useful tool in recorded not only if brushing had taken place but also the resident's preference over a type of toothbrush or if anything looked different such as a sore patch or ulcer. This can become a vital tool in identifying concerns, which may be missed such as early oral cancer lesions. This was a recommendation, daily oral care plans, by the Smiling Matters CQC report. This is a recommendation in the NICE guidance, which states that oral health assessments and mouth care plans should be performed along with daily mouth care and recording keeping, integrating them with the ADL, means that this is a more holistic or person-centered approach to patient care. ${ }^{5,12}$ The NICE recommendations also identify a need for care staff training, this research has identified a need for residents with capacity and their family members to increase awareness of the need for good oral health and hygiene and the impacts on general health.

\section{Limitations}

This study was performed on a very small sample, both the care home and dental practice engaged with a good standard with CQC ratings that reflected the high standard of patient care they delivered. The area had good transport links meaning that physical access to services was easier than in some other localities and the care home had both local authority and privately funded patients. The dental practice was for private patients and Denplan only. This could impact the finding of the research and they may not be transferable to other more rural areas or if the research methods were repeated in a poor-quality care home or large NHS dental practice.

\section{Conclusion and Recommendations}

Similar themes were identified across the three cohorts. Capturing the voice of the residents living in a care home, with or without dementia, was challenging, the friends and family members were happy to speak alongside the residents. Challenges around transport and access were raised but all this was rooted in the fact that dental health was seen as less important than medical or holistic health, and frequently, dentistry was a responsive service rather than a preventive service. Education across the care home staff and residents, and friends and family could go some way to addressing this. The recommendations based on the findings from this research are:

- Better consistent training for care home professionals.

- Information for care home residents and their families on the cost of dental treatment and the importance of good oral hygiene.
- Support on traveling to dental appointments.

- Routine oral health care plans and daily tick sheets to support those who need reminding or assistance in carrying out daily oral hygiene routines.

- Pathway in place for every resident on arrival at the care home to continue care with their dentist or to book an appointment with a local dental care provider.

\section{Conflict of Interest}

None declared.

\section{References}

1 Care Quality Commission. The state of health care and adult social care in England 2014/15. 2015. Available at: https:// www.cqc.org.uk/sites/default/files/20151013_CQC_State_of_ Care_Report_WEB.pdf. Accessed July 17, 2019

2 Public Health England. Public health profiles- Essex. 2019. [Online] Available at: https://fingertips.phe.org.uk. Accessed July 17, 2019

3 Alzheimer's Society. Dementia overview. 2016. Available at: https://www.alzheimers.org.uk/info/20025/policy_and_influencing/251/dementia_uk. Accessed April 23, 2018

4 Department of Health. Living well with dementia- a national strategy. 2009. Available at: https://www.alzheimers.org. uk/info/20025/policy_and_influencing/251/dementia_uk. Accessed April 23, 2018

5 NICE. Dementia overview flow chart. 2018. Available at: https://pathways.nice.org.uk/pathways/dementia. Accessed April 23, 2018

6 Ueda K. Preventing aspiration pneumonia by oral health care. Japan Med Assoc J 2011;54(1):39-43

7 Eaton KA, Lloyd HA, Wheeler M, et al. Looking after the mouth - evaluation of a pilot for a new approach to training care home carers in Kent, Surrey and Sussex. Br Dent J 2016;221(1):31-36

8 Public Health England. What is known about the oral health of older people in England and Wales: a review of oral health surveys of older people. 2015. Available at: https://www.gov. uk/government/uploads/system/uploads/attachment_data/ file/489756/What_is_known_about_the_oral_health_of_ older_people.pdf. Accessed April 23, 2018

9 Essex County Council. Living well with dementia- A dementia strategy for Essex, Southend and Thurrock living well Essex. 2017. Available at: https://www.livingwellessex.org/ media/523329/Dementia-Strategy.pdf. Accessed April 23, 2018]

10 Public Health England. Delivering better oral health: An evidence-based toolkit for prevention. 2017. Available at: https://www.gov.uk/government/publications/delivering-better-oral-health-an-evidence-based-toolkit-for-prevention. Accessed July 18, 2019

11 Ritchie J, Lewis J, Nicholls CM, Ormston R. Qualitative Research Practice : A Guide for Social Science Students and Researchers. 2nd ed. SAGE Publications Ltd; 2013

12 Care Quality Commission. Smiling matters: Oral health care in care homes. 2019. Available at: https://www.cqc.org.uk/ sites/default/files/20190624_smiling_matters_full_report.pdf. Accessed April 23, 2018 